gange gemessen. Diese Versuche ergaben, dass unter dem Einflusse kalter Wannen das Volumen des Gehirns zunahm und zu gleicher Zeit Temperaturerniedrigung im äusseren Gehörgange eintrat, wie auch in den vorhergehenden Versuchen. Die entgegengesetzte Erscheinung erwies sich bei heissen Handwannen. Auf Grund dieser, obgleich nicht zahlreichen, dabei aber keine Ausnahme bildenden Versuche geht hervor, dass der äussere Gehörgang sich zu thermischen Reizungen ganz ebenso verhält, wie die peripherischen Körpertheile, und dass die Temperatur der peripherischen Theile bei denselben Reizungen im entgegengesetzten Verhältnisse zu den Gehirngefässen steht.

(Aus dem physiologischen Institut der Cniversität Strassburg.)

\title{
Versuche über die Durchschneidung der hinteren Gehirncommissur beim Kaninchen.
}

Yon

1. Darkschewitsch aus Moskau.

Hierzu Tafel II.

Bei der Untersuchung des Kaninchengehirns konnte ich mich davon überzeugen, dass jene Fasern („ventrale Partie $\left.{ }^{4}\right)^{1}$ ) der hinteren Gehirncommissur, welche in enger Beziehung sowohl zum Oculomotoriuskerne, wie auch vermittelst des hinteren Längsbündels zu den Kernen anderer motorischer Augennerven stehen, in der Glandula pinealis, dem Ganglion habenulae und zum Theil auch in der Hirnrinde endigen. Andererseits fand ich, dass sich ein Theil der Fasern vom gemeinsamen Stamme des Tractus opticus

1) VgI. "Ueber die hintere Commissur des Gehirns". Neurologisches Centralblatt, 1885, $\mathrm{Nr}, 5$. 
anf dem Wege zum oberen Vierhïgel abzweigt, durch die Sub$\operatorname{stanz}$ des Corp. geniculatum ext. in der Richtung nach dem Ganglion habenulae hindurchgeht und zum Theil in diesem letzteren sein Ende findet, zum Theil aber sich den Fasern des Pedunculus conarii anschliesst; um gemeinschaftlich mit denselben nach der Glandula pinealis zu verlaufen ${ }^{1}$ ). Die beigefügte Fig. I stellt das auf Grund meiner Präparate entworfene Schema der beschriebenen Verhältnisse dar. $c$ und $c^{\prime}$ bedeuten die Fasern der hinteren Commissur, die nach dẹ Kreuzung in der Glandula pinealis $(a)$ und in dem Ganglion habenulae $\left(b, b^{\prime}\right)$ endigen; $d$ und $d^{\prime}$ sind die Fasern der Tractus optici, welche nach ihrer Trennung von den Stämmen $(e, e)$ nunmehr theils nach dem Ganglion habenulae, theils nach der Glandula pinealis hinziehen.

Die Ergebnisse der histologisehen Forschung sprachen demnach sehr zu Gunsten der Ausicht, dass in der hinteren Commissur (in ihrer ventralen Partie) die Bahn zu suchen sei, durch welche Reize vom Tractus opticus auf den Nervus oculomotorius übergehen können. Aus diesem Grunde stellte ich mir die Aufgabe, auf experimentellem Wege zu bestimmen, welchen Einfluss die Zerstörung der hinteren Commissur auf die Pupillenreaction ausiibt. Ich stellte daher eine Reihe von Versuchen an, bei denen ich die hintere Gehirneommissur bei Kaninchen durchschnitt.

Die Operation wurde folgendermaassen ansgeführt: Es wurden einem Kaninchen in der Aethernarcose die Hautdecken des Schädels durch einen Schnitt längs der Sutura longitudinalis geöffnet. 11/2 bis $2 \mathrm{~mm}$ nach vorn vom Tuberculum interparietale (W. Krause) wurde in den Knochen mit Hülfe des Trepans eine Oeffnung gemacht und dieselbe darauf mittelst der Knochenzange in der Länge um $15 \mathrm{~mm}$, in der Quere um $11 \mathrm{~mm}$ erweitert. Die Dura mater wurde zu beiden Seiten des Sinus longitudinalis parallel den Rändern der Knochenöffnung eingeschnitten, so dass zwei Lappen entstanden, die mit einander durch den Sinus zusammenhingen. Darauf wurde in den Zwischenraum zwischen beiden Hemisphären rechts oder links vom Sinus longitudinalis an einer Stelle, welche ungefähr der vorderen Partie des oberen Vierhügels entsprechen musste, ein schmales spitzes Messer eingeführt. An der Schneide des Messers befand sich eine Marke, die ich mir fruher in der

1) Noch nioht veröffentlicht. 
richtigen Entfernung von der Spitze vorgezeichnet hatte, um nur die hintere Commissur durchzuschneiden, tiefer liegende Theile jedoch nicht mit zu verletzen. Nach dem Einstechen wurde das Messer vorsichtig parallel dem Sinus nm $1 \frac{1}{2} / 2-2 \mathrm{~mm}$ vorgeschoben und dann entfernt. Die Lappen der Hirnhaut wurden auf der Hirnoberfläche zurechtgelegt und darauf die Wundränder durch eine gewöhnliche Naht vereinigt. Die Blutung, welche beim Einstechen des Messers auftritt, ist in der Regel nicht heftig und steht bald, nachdem die Wunde vernäht ist. Die Eröffnung der Dura in der eben beschriebenen Weise ist unseres Erachtens nach äusserst wichtig, denn durch sie wird einer übermässigen Compression des Gehirns durch das Blut vorgebeugt, welches letztere sich sonst unter der Hirnhaut ansammelt und ohne genügenden Abfluss auf das Gehirn stark zu driicken anfängt. Ausserdem klemmt sich, falls in der Hirnhaut nur ein Einschnitt gemacht wird, die Substanz des Gehirns nicht selten in demselben ein und wird nekrotisch.

Die beschriebene Operation vertragen die Kaninchen sehr leicht and schon in ein bis zwei Stunden erholen sie sich so weit, dass sie wieder Futter zu sich nehmen. Die Heilung der Haut geht ziemlich scbnell vor sich und immer per primam intentionem, wobei es gar keiner besonderen Pflege bedarf. Bei manchen Kaninchen war zwar unter den Hautdecken eine ziemlich erhebliche Anhäufung käsiger Massen zu bemerken, allein auch in solchen Fällen sah ich einen relativ besseren Heilungsverlauf, wenn ich die Wunde vollständig sich selbst tuberliess, als wenn ich die käsigen Massen "entfernte.

Um das Ergebniss der Operation - die Veränderung in der Pupillenbeweglichkeit nach Durehschneidung der hinteren Commissur - beurtheilen zu können, stelite ich vor der Operation für jedes einzelne Kaninchen die Grenzen der Contractilität seiner Pupillen bei Lichtreizen fest. $\mathrm{Zu}$ diesem Zwecke benutzte ich Gas- und Sonnenlicht. Im ersteren Falle wurde das Licht einer Gaslampe mittelst einer planconvexen Linse in einem sonst ganz dunklen Raume anf die Augen geworfen, im letzteren Falle wurde das Kaninchen in einem gewöhnlichen gebracht, dass in seine beiden Augen directes Sonnenlicht hineinfiel. In beiden Fällen wurde mit Hülfe eines in Millimeter eingetheilten Maassstabes der kleinste Durchmesser der Pupillen des 
betreffenden Kaninchens notirt und zwar 1) wenn das Licht in beide Augen fiel und 2) wenn das Licht ein Auge beleuchtete, während das andere Auge verdeckt blieb. Nach der Operation wurde dann die Pupille in eben derselben Weise auf ihre Beweglichkeit hin untersucht. Auch in diesem Falle notirte ich in Millimetern den kleinsten Durchmesser der Pupillen 1) bei gleichzeitiger Beleuchtung beider Augen, 2) bei Beleuchtung des einen Auges, während das andere verdeckt blieb und 3) wenn der Lichtreiz nicht das zu untersuchende, sondern das andere Auge traf.

Bei der Schwierigkeit der Operation ist es selbstverständlich, dass sie nicht immer glïckte. In der That kamen bei Ausführung der Operation häufig Abweichungen vor, die das Ergebniss der ganzen Operation wesentlich anders gestalteten. Neben den Fällen, wo die Operation gewisse Veränderungen in der Pupillenbeweglichkeit nach sich zog, gab es auch solche, wo die Operation ohne jeden Einfluss auf die Iriscontraction blieb. Dieser Umstand veranlasste mich einen jeden Fall nach der Section möglichst eingehend, nicht nur makroskopisch, sondern auch mikroskopisch zu untersuchen. Ich hielt es ausserdem für wichtig, diejenigen Kaninchen, bei welchen ich nach der Operation eine gewisse Veränderung in der Beweglichkeit der Pupillen beobachtete, möglichst lange am Leben zu lassen, um mich von der Beständigkeit der auf die Operation folgenden Erscheinungen zu überzeugen. Deshalb tödtete ich die Kaninchen, bei welchen die Pupillenbeweglichkeit sich nach der Operation normal erwies, schon im Laufe der ersten Woche, dagegen diejenigen, bei welchen die Operation irgend eine Veränderung in der Irisbeweglichkeit nach sich zog, erst nach 3 bis 9 Wochen.

Es wurden im Ganzen 20 Kaninchen operirt, davon starben 3 im Laufe der ersten 36 Stunden infolge eines Blutergusses in's Kopfmark (das Messer war durch die Substantia perforata posteria gedrungen), bei 4 wurden keinerlei Störungen in der Bewegung der Pupille wahrgenommen, bei 13 sind versehiedene Veränderungen in der Beweglichkeit der Iris aufgetreten.

Bei der speciellen Beschreibung der Resultate anserer Operationen wollen wir dieselben in zwei Abtheilungen gruppiren, die sich durch die verschiedenen Papillenreactionen charakterisiren. 


\section{Erste Gruppe.}

\begin{tabular}{|c|c|c|c|c|}
\hline \multirow{2}{*}{$\begin{array}{c}\text { Kaninchen No. 3. Durchmesser } \\
\text { der engsten Pupille. }\end{array}$} & \multicolumn{2}{|c|}{ Vor der Operation } & \multicolumn{2}{|c|}{ Nach der Operation } \\
\hline & R. Auge & L. Auge. & R. Auge. & L. Auge. \\
\hline 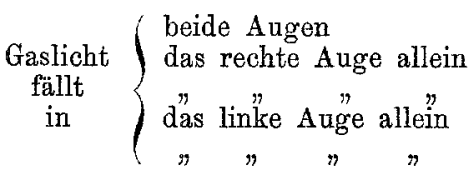 & $\begin{array}{l}4 \\
4 \\
- \\
-\end{array}$ & $\frac{4}{4}$ & $\frac{4}{4}$ & $\begin{array}{l}\frac{4}{4} \\
4 \\
-\end{array}$ \\
\hline $\begin{array}{c}\text { Sonnen- } \\
\text { licht } \\
\text { fällt in }\end{array}\left\{\begin{array}{l}\text { beide Augen } \\
\text { das rechte Auge allein } \\
\text { das linke Auge allein } \\
" \Rightarrow " ~\end{array}\right.$ & $\begin{array}{l}21 / 2 \\
21 / 2 \\
- \\
-\end{array}$ & $\frac{21 / 2}{\overline{21 / 2}}$ & $\begin{array}{l}2^{1 / 2} \\
2^{1 / 2} / 2 \\
\overline{-} \\
2^{1} / 2\end{array}$ & $\begin{array}{l}\frac{21 / 2}{21 / 2} \\
21 / 2 \\
-\end{array}$ \\
\hline
\end{tabular}

Operirt den 13./5., secirt den 18./5. Befund: Durch einen Längsschnitt ist die hintere Partie des oberen und die vordere Partie des unteren Vierhügels seitlich von der Mittellinie verletzt (Fig. III, 8). Die hintere Commissur ist intact.

\begin{tabular}{|c|c|c|c|c|}
\hline \multirow{2}{*}{$\begin{array}{c}\text { Kaninchen No. 4. Durchmesser } \\
\text { der engsten Pupille. }\end{array}$} & \multicolumn{2}{|c|}{ Vor der Operation } & \multicolumn{2}{|c|}{ Nach der Operation } \\
\hline & R. Auge. & L. Auge. & R. Auge. & L. Auge. \\
\hline $\begin{array}{c}\text { Gaslicht } \\
\text { fällt } \\
\text { in }\end{array}\left\{\begin{array}{l}\text { beide Augen } \\
\text { das rechte Auge allein } \\
\text { das linke Auge allein } \\
" \# " \Rightarrow "\end{array}\right.$ & $\begin{array}{l}31 / 2 \\
3^{1 / 2} \\
- \\
-\end{array}$ & $\begin{array}{l}3^{1 / 2} \\
\overline{3^{1}} / 2 \\
-\end{array}$ & $\begin{array}{l}3^{1} / 2 \\
3^{1} / 2 \\
\overline{31 / 2}\end{array}$ & $\begin{array}{l}3^{1 / 2} \\
\overline{31 / 2} \\
3^{1 / 2} / 2 \\
-\end{array}$ \\
\hline $\begin{array}{c}\text { Sonnen- } \\
\text { licht } \\
\text { fällt in }\end{array}\left\{\begin{array}{l}\text { beide Augen } \\
\text { das rechte Auge allein } \\
\text { das linke Auge allein } \\
" \# " \Rightarrow "\end{array}\right.$ & $\begin{array}{l}2 \\
2 \\
- \\
-\end{array}$ & $\begin{array}{l}2 \\
- \\
2 \\
-\end{array}$ & $\begin{array}{l}2 \\
2 \\
- \\
2\end{array}$ & $\begin{array}{l}\frac{2}{2} \\
2 \\
-\end{array}$ \\
\hline
\end{tabular}

Operirt den 15./5. Nach der Operation bewegt sich das Kaninchen beständig nach rückwärts; zu sitzen vermag es nicht. Secirt den 16./5. Befund: Durch einen Längsschnitt ist die hintere Partie des oberen Vierhügels, der gesammte hintere Vierhügel und die vordere Partie des Vermis cerebelli in der Mittellinie verletzt (Fig. III, 6). Die hintere Commissur ist intact. 
Versuche üb.d. Durchschneidung d. hinteren Gehirncommissur b. Kaninchen. 125

\begin{tabular}{|c|c|c|c|c|}
\hline \multirow{2}{*}{$\begin{array}{c}\text { Kaninchen No. 6. Durchmesser } \\
\text { der engsten Pupille. }\end{array}$} & \multicolumn{2}{|c|}{ Vor der Operation } & \multicolumn{2}{|c|}{ Nach der Operation } \\
\hline & R. Auge. & L. Auge. & R. Auge. & L. Auge. \\
\hline $\begin{array}{c}\text { Gaslicht } \\
\text { fällt } \\
\text { in }\end{array}\left\{\begin{array}{l}\text { beide Augen } \\
\text { das rechte Auge allein } \\
\text { das linke Auge allein } \\
" n " \Rightarrow "\end{array}\right.$ & $\begin{array}{l}5 \\
5 \\
- \\
-\end{array}$ & $\frac{5}{5}$ & $\begin{array}{l}5 \\
5 \\
- \\
5\end{array}$ & $\frac{5}{5}$ \\
\hline $\begin{array}{c}\text { Sonnen- } \\
\text { licht } \\
\text { fällt in }\end{array}\left\{\begin{array}{l}\text { beide Augen } \\
\text { das rechte Auge allein } \\
\text { das linke Auge allein } \\
" \# " " \Rightarrow\end{array}\right.$ & $\begin{array}{l}3 \\
3 \\
- \\
-\end{array}$ & $\frac{3}{3}$ & $\begin{array}{l}3 \\
3 \\
\\
3\end{array}$ & $\frac{3}{3}$ \\
\hline
\end{tabular}

Operirt den 18./5. Secirt den 21./5. Befund: Die Commissura mollís ist in der Mittellinie verletzt (Fig. III, 2). Die hintere Commissur ist intact.

\begin{tabular}{|c|c|c|c|c|}
\hline \multirow{2}{*}{$\begin{array}{c}\text { Kaninchen No. 13. Durchmesser } \\
\text { der engsten Pupille. }\end{array}$} & \multicolumn{2}{|c|}{ Vor der Operation } & \multicolumn{2}{|c|}{ Nach der Operation } \\
\hline & R. Auge. & L. Auge. & R. Auge. & L. Auge. \\
\hline $\begin{array}{c}\text { Gaslicht } \\
\text { fällt } \\
\text { in }\end{array}\left\{\begin{array}{l}\text { beide Augen } \\
\text { das rechte Aage allein } \\
\text { das linke Auge allein } \\
" \quad " \quad " \quad "\end{array}\right.$ & $\begin{array}{l}5 \\
5 \\
- \\
-\end{array}$ & $\begin{array}{l}51 / 2 \\
-- \\
51 / 2 \\
-\end{array}$ & $\begin{array}{l}5 \\
\overline{5} \\
\overline{5}\end{array}$ & $\begin{array}{l}5^{1 / 2} \\
\overline{5^{1 / 2}} \\
5^{1 / 2} \\
-\end{array}$ \\
\hline $\begin{array}{c}\text { Sonnen- } \\
\text { licht }\end{array}\left\{\begin{array}{l}\text { beide Augen } \\
\text { das rechte Auge allein } \\
\text { fält in }\end{array}\left\{\begin{array}{c}" \text { das linke Auge allein } \\
" \# " \# \Rightarrow "\end{array}\right.\right.$ & $\begin{array}{l}3 \\
3 \\
- \\
-\end{array}$ & $\frac{31 / 2}{\overline{31 / 4}}$ & $\begin{array}{l}3 \\
\frac{3}{3} \\
3\end{array}$ & $\begin{array}{l}31 / 4 \\
3^{1 / 4} \\
3^{1 / 4} \\
-\end{array}$ \\
\hline
\end{tabular}

Operirt den 29./5. Zwangshaltung des Kopfes und Zwangsbewegungen sind nicht vorhanden. Secirt den 5./6. Befund: Die Commissura mollis ist durch einen Längsschnitt links von der Mittellinie, parallel der Taenia thalamie optici verletzt (Fig. III, 2). Die hintere Commissur ist intact. 


\section{Zweite Gruppe.}

A.

\begin{tabular}{|c|c|c|c|c|}
\hline \multirow{2}{*}{$\begin{array}{c}\text { Kaninchen Nr. 2. Durchmesser } \\
\text { der engsten Pupille. }\end{array}$} & \multicolumn{2}{|c|}{ Vor der Operation } & \multicolumn{2}{|c|}{ Nach der Operation } \\
\hline & R. Auge. & L. Auge. & R. Auge. & L. Auge. \\
\hline $\begin{array}{c}\text { Gaslicht } \\
\text { fällt } \\
\text { in }\end{array}\left\{\begin{array}{l}\text { beide Augen } \\
\text { das rechte Auge allein } \\
\text { das linke Auge allein } \\
" \Rightarrow " \Rightarrow "\end{array}\right.$ & $\begin{array}{l}6 \\
6 \\
- \\
- \\
-\end{array}$ & $\frac{6}{\frac{6}{6}}$ & $\begin{array}{c}10^{1} / 2 \\
10^{1 / 2} / 2 \\
- \\
10^{1 / 2}\end{array}$ & $\begin{array}{l}10^{1} / 2 \\
\overline{101} / 2 \\
10^{1} / 2 \\
-\end{array}$ \\
\hline $\begin{array}{c}\text { Sonnen- } \\
\text { licht } \\
\text { fällt in }\end{array}\left\{\begin{array}{l}\text { beide Augen } \\
\text { das rechte Auge allein } \\
\text { das linke Auge allein } \\
" \Rightarrow " \#\end{array}\right.$ & $\begin{array}{l}3 \\
3 \\
- \\
-\end{array}$ & $\frac{3}{\frac{3}{3}}$ & $\begin{array}{l}71 / 2 \\
71 / 2 \\
- \\
71 / 2\end{array}$ & $\begin{array}{l}71 / 2 \\
\overline{71} / 2 \\
71 / 2 \\
-\end{array}$ \\
\hline
\end{tabular}

Operirt den 9./5. Secirt den 12./7. Bei makroskopischer Untersuchung ergiebt sich: der obere Vierhügel ist durch einen Längsschnitt in der Mittellinie verletzt (Fig. VI, 1). Verletzung der Glandula pinealis.

Nach Erhärtung des Gehirns in doppeltehromsaurem Kali wurden Schnitte in frontaler Ebene angefertigt. Die Fig. II stellt einen solchen Schnitt dar, welcher aus dem Niveau $b$ des Gehirns (Fig. VI) entnommen ist. Bei Untersuchung der Präparate ergiebt sich Folgendes:

1. Das Messer war nur bis zur Höhle des Aquaeductus sylvii gedrungen, ohne die tiefer liegenden Theile verletzt zu haben (Fig. II); es sind also die Kerne mit den Oculomotoriuswurzeln und der Boden des dritten Ventrikels intact geblieben $\mathbf{1}$ ).

2. Die hintere Commissur ist in der Mittellinie und zwar nur in ihrem hinteren Abschnitte, aber fast total zerstört (Fig. II); jhre vordere Partie ist intact.

3. Ein Theil der Substanz des oberen Vierhügels ist in der Mittellinie and vorzüglich in seinem hinteren Abschnitte zerstört (Fig. II).

1) Unter "Boden des dritten Ventrikels" verstehen wir den Boden des Grübchens, welches lateral von der inneren Seite der Thalami optici, vorn von der Commissura mollis und hinten von der hinteren Commissur begrenzt wird. 


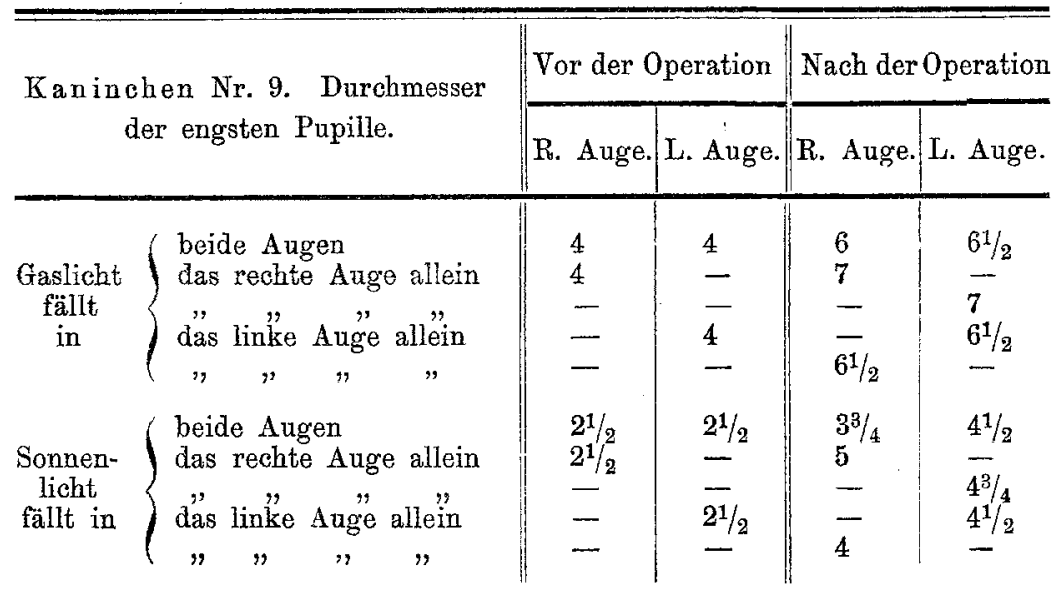

Operirt den 20./5. Nach der Operation wird eine Zwangslage des Kopfes beobachtet: die linke Seite desselben ist nach oben, die rechte nach unten gedreht. Secirt den 13./6. Befund bei makroskopischer Untersuchung des Gehirns: Durch einen Längsschnitt ist die hintere Commissur, die linke hintere seitliche Wand des dritten Ventrikels ${ }^{1}$ ) und der hintere Abschnitt der Commissura mollis verletzt (Fig. VI, 2). Verletzong der Glandula pinealis.

Nach Erhärtung des Gehirns in doppeltchromsaurem Kali wurden Schnitte in frontaler Ebene gemacht. Bei Untersuchung der letzteren ergiebt sich:

1. Die hintere Commissur ist seitlich (links) von der Mittellinie durchsehnitten (Fig. IV, 6).

2. Der Boden des dritten Ventrikels ist seitlich (links) von der Mittellinie verletzt (Fig. IV, 7).

3. Die Kerne und Wurzeln des Oculomotorius sind intact.

4. In der Substanz des oberen Vierhügels ist kein Defect vorhanden.

1) Unter "hintere seitliche Wand" des dritten Veatrikels verstehen wir die Wand des dritten Ventrikels zwischen Comm. mollis und Comm, posterior. 


\begin{tabular}{|c|c|c|c|c|}
\hline \multirow{2}{*}{$\begin{array}{c}\text { Kaninchen No. } 14 . \text { Durchmesser } \\
\text { der engsten Pupille. }\end{array}$} & \multicolumn{2}{|c|}{ Vor der Operation } & \multicolumn{2}{|c|}{ Nach der Operation } \\
\hline & R. Auge & L. Auge. & R. Auge. & L. Auge. \\
\hline $\begin{array}{c}\text { Gaslicht } \\
\text { fällt } \\
\text { in }\end{array}\left\{\begin{array}{l}\text { beide Augen } \\
\text { das rechte Auge allein } \\
\text { das linke Auge allein } \\
", " \Rightarrow ",\end{array}\right.$ & $\begin{array}{l}4^{1 / 2} \\
4^{1 / 2} \\
- \\
-\end{array}$ & $\frac{4}{2}$ & $\begin{array}{l}6 \\
6 \\
- \\
6\end{array}$ & $\begin{array}{l}\frac{51 / 2}{51 / 2} \\
5^{1 / 2}\end{array}$ \\
\hline $\begin{array}{l}\text { Sonnen- } \\
\text { licht } \\
\text { fällt in }\end{array}\left\{\begin{array}{l}\text { das rechte Auge allein } \\
\text { das linke Auge allein } \\
" \text { " " " " }\end{array}\right.$ & $\begin{array}{l}31 / 4 \\
31 / 4 \\
- \\
-\end{array}$ & $\begin{array}{l}3 \\
-- \\
- \\
-\end{array}$ & $\begin{array}{l}4^{1 / 2} / 2 \\
41 / 2 \\
- \\
41 / 2\end{array}$ & $\begin{array}{l}\frac{41 / 4}{41 / 4} \\
4^{1} / 4\end{array}$ \\
\hline
\end{tabular}

Operirt den 3./6. Nach der Operation besteht keine Zwangslage des Kopfes. Secirt den 8./7. Bei makroskopischer Untersuchung des Gehirns ergiebt sich: durch einen Längsschnitt ist die vordere Partie der hinteren Commissur, der Boden des dritten Ventrikels und der hintere Abschnitt der Commissura mollis verletat (Fig. VI, 3). Die Glandvla pinealis ist in ihrem vorderen Theile verletzt.

Bei mikroskopischer Untersuchung von in frontaler Ebene gemachten Schnitten wurde gefunden:

1. Die hintere Commissur ist in ihrem vorderen Theile ]ängs der Mittellinie zerstört.

2. Der Boden des dritten Ventrikels ist in der Mittellinie verletzt.

3. Die Kerne und Wurzeln des Oculomotorius sind intact.

4. Ein Defect in der Substanz des oberen Vierhügels ist nicht vorhanden.

\begin{tabular}{|c|c|c|c|c|}
\hline \multirow{2}{*}{$\begin{array}{c}\text { Kaninchen No. } 15 . \text { Durchmesser } \\
\text { der engsten Pupille. }\end{array}$} & \multicolumn{2}{|c|}{ Vor der Operation } & \multicolumn{2}{|c|}{ Nach der Operation } \\
\hline & R. Auge. & L. Auge. & R. Auge. & L. Auge. \\
\hline $\begin{array}{c}\text { Gaslicht } \\
\text { fällt } \\
\text { in }\end{array}\left\{\begin{array}{l}\text { beide Augen } \\
\text { das rechte Auge allein } \\
\text { das linke Auge allein } \\
" \Rightarrow " ", "\end{array}\right.$ & $\begin{array}{l}5 \\
5 \\
- \\
-\end{array}$ & $\frac{5}{5}$ & $\begin{array}{l}8 \\
8^{1 / 2} \\
- \\
-\end{array}$ & $\begin{array}{l}\frac{7}{7} \\
8 \\
-\end{array}$ \\
\hline $\begin{array}{c}\text { Sonnen- } \\
\text { licht } \\
\text { fällt in }\end{array}\left\{\begin{array}{l}\text { beide Augen } \\
\text { das rechte Auge allein } \\
\text { das linke Auge allein } \\
", ", " ~\end{array}\right.$ & $\begin{array}{l}3 \\
3 \\
- \\
- \\
-\end{array}$ & $\frac{3}{3}$ & $\begin{array}{l}7^{1 / 2} \\
- \\
- \\
71 / 2\end{array}$ & $\begin{array}{l}6^{1 / 2} \\
6^{1 / 2} \\
7 \\
-\end{array}$ \\
\hline
\end{tabular}


Versuche üb. d. Durchschneidung d. hinteren Gehirncommissur b. Kaninchen. 129

Operirt den 2./6. Gleich nach der Operation linksseitige Zwangslage. Secirt den 12./7. Die makroskopische Untersuchung ergiebt: durch einen Längsschnitt ist die hintere Commissur, die Glandula pinealis und die rechte hintere seitliche Wand des dritten Ventrikels verletzt.

Die mikroskopische Untersuchung von in frontaler Ebene gemachten Schnitten zeigt:

1. Verletzung der hinteren Commissur in der Mittellinie.

2. Der Boden des dritten Ventrikels ist intact.

3. In der.Substanz des oberen Vierhügels ist kein Defect vorhanden.

4. Ein Theil des rechten Oculomotoriuskernes ist durch einen Bluterguss zerstört.

\begin{tabular}{|c|c|c|c|c|}
\hline \multirow{2}{*}{$\begin{array}{c}\text { Kaninchen No. } 10 . \text { Durchmesser } \\
\text { der engsten Pupille. }\end{array}$} & \multicolumn{2}{|c|}{ Vor der Operation } & \multicolumn{2}{|c|}{ Nach der Operation } \\
\hline & R. Auge & L. Auge. & R. Auge. & L. Auge. \\
\hline $\begin{array}{c}\text { Gaslicht } \\
\text { fällt } \\
\text { in }\end{array}\left\{\begin{array}{l}\text { beide Augen } \\
\text { das rechte Auge allein } \\
\text { das linke Auge allein } \\
" \text { " " " } "\end{array}\right.$ & $\begin{array}{l}4 \\
4 \\
- \\
-\end{array}$ & $\begin{array}{l}4 \\
\frac{4}{4} \\
-\end{array}$ & $\begin{array}{l}5 \\
51 / 2 \\
\frac{5}{5}\end{array}$ & $\begin{array}{l}\frac{5^{1 / 2}}{6} \\
5^{1 / 2}\end{array}$ \\
\hline $\begin{array}{c}\text { Sonnen- } \\
\text { licht } \\
\text { fällt in }\end{array}\left\{\begin{array}{l}\text { beide Augen } \\
\text { das rechte Auge allein } \\
\text { das linke Auge allein } \\
", ", ", "\end{array}\right.$ & $\begin{array}{l}21 / 2 \\
2^{1 / 2} \\
- \\
-\end{array}$ & $\begin{array}{l}21 / 2 \\
- \\
- \\
-\end{array}$ & $\begin{array}{l}3^{1 / 2} \\
4^{3} / 4 \\
- \\
- \\
3^{1} / 2\end{array}$ & $\begin{array}{l}4^{3 / 4} \\
5 \\
4^{3 / 4} \\
-\end{array}$ \\
\hline
\end{tabular}

Operirt den 22./5. Gleich nach der Operation Zwangslage des Kopfes: die linke Seite desselben ist nach oben gedreht. Secirt den 24./6. Bei makroskopischer Untersuchung des Gebirns ergiebt sich: durch einen Längsschnitt ist die hintere Commissur seitlich (links) von der Mittellinie verletzt; der Pedunculus conarii sinister ist durchschnitten; die linke hintere seitliche Wand des dritten Ventrikels ist verletzt (ein der Fig. VI, 2 analoger Fall).

Die mikroskopische Untersuchung von in frontaler Ebene angefertigten Schnitten ergiebt:

1. Der Schnitt war seitlich von der hinteren Commissur gefuihrt (Fig.V,7).

2. Der Boden des dritten Ventrikels ist seitlich (links) von der Mittellinie verletzt (Fig. V, 7).

3. In der Substanz des oberen Vierhügels ist kein Defect vorhanden.

4. Die Kerne und Wurzeln des Oculomotorius sind intact.

Kaninchen Nr. 7. Die Resultate der Operation sind den von Nr. 9 analog. 
B.

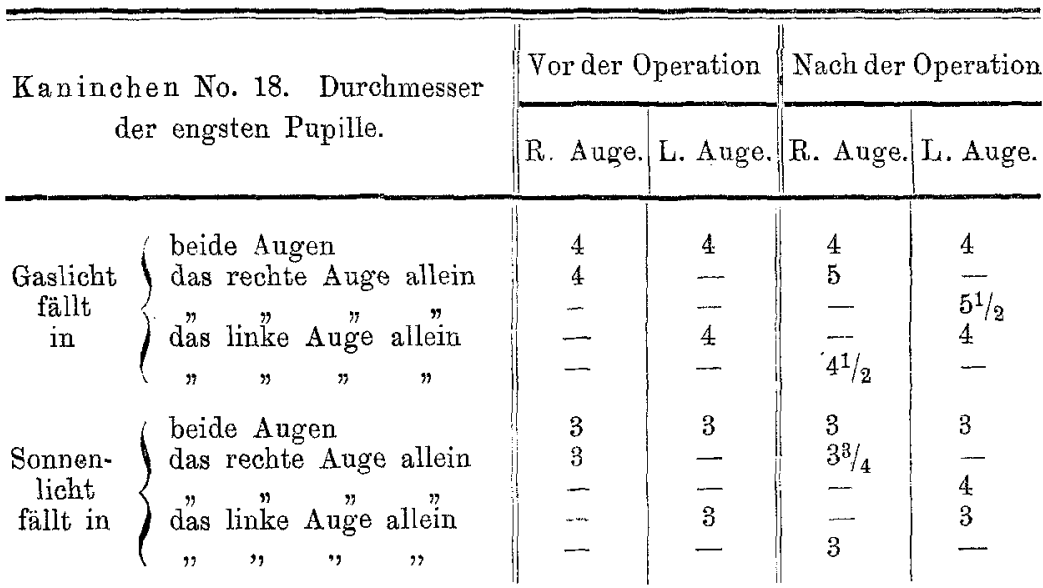

Operirt den 15.6. Gleich nach der Operation macht das Kaninchen, zum Laufen veranlasst, Kreisbewegungen nach der rechten Seite hin. Secirt den 25./6. Die makroskopische Untersurhurg des Gehirns ergiebt: Verletzung der hinteren Partie des Thatamus opticus sinister und des Corpus geniculatum ext. sinister durch einen Längsschnitt, der von aussen und parallel der Taenia thalami optici verläuft (Fig. VI, 4). Die hintere Commissur und die Glandula pinealis sind intact.

\begin{tabular}{|c|c|c|c|c|}
\hline \multirow{2}{*}{$\begin{array}{c}\text { Kaninchen No. 5. Durchmesser } \\
\text { der engsten Pupille. }\end{array}$} & \multicolumn{2}{|c|}{ Vor der Operation } & \multicolumn{2}{|c|}{ Nach der Operation } \\
\hline & R. Auge. & I. Augge. & P. Auge. & L. Auge. \\
\hline $\begin{array}{c}\text { Gaslicht } \\
\text { fällt } \\
\text { in }\end{array}\left\{\begin{array}{l}\text { beide Augen } \\
\text { das rechte Auge allein } \\
\text { das linke Auge allein } \\
" \Rightarrow " n "\end{array}\right.$ & $\begin{array}{l}61 / 2 \\
61 / 2 \\
- \\
-\end{array}$ & $\frac{61 / 2}{6^{1 / 2}}$ & $\begin{array}{l}61 / 2 \\
7 \\
- \\
61 / 2\end{array}$ & $\begin{array}{l}61 / 2 \\
7 \\
61 / 2\end{array}$ \\
\hline $\begin{array}{l}\text { Sonnen- } \\
\text { licht } \\
\text { fällt in }\end{array}\left\{\begin{array}{l}\text { beide Augen } \\
\text { das rechte Auge allein } \\
\text { das linke Auge allein } \\
" \| " n " ~\end{array}\right.$ & $\begin{array}{l}4^{1 / 2} \\
4^{1 / 2} \\
- \\
-\end{array}$ & $\begin{array}{l}4^{1 / 2} \\
-\overrightarrow{41 / 2}\end{array}$ & $\begin{array}{l}4^{1 / 2} \\
5 \\
- \\
4^{1 / 2}\end{array}$ & $\begin{array}{l}41 / 2 \\
5 \\
4^{1 / 2} \\
-\end{array}$ \\
\hline
\end{tabular}

Operirt den 16./5. Gleich nach der Operation macht sich eine Zwangslage des Kopfes bemerkbar: die linke Seite desselben ist nach oben gedreht. Secirt den 19./6. Die makroskopische Untersuchung des Gehirns ergiebt: eine leichte Verletzung der linken hinteren seitlichen Wand des dritten Ventrikels and des Pedunculus conarii sinister (Fig. III, 3).

Kaninchen Nr.12. Die nach der Operation aufgetretenen Erscheinungen sind denjenigen, die beim Kaninchen Nr. 5 beobachtet wurden, analog. 
Versuche üb. d. Durchschneidung d. hinteren Gehirncommissur b. Kaninchen. 131

II.

Bevor wir an die Analyse der Resultate unserer Versuche herantreten, wollen wir einige Worte über die Untersuchung der Pupillencontractilität sagen.

Die Beweglichkeit der Iris ist bei Kaninchen sehr träge und man kann nicht selten auf eine Contractilität der Pupille nur ans genauer Messung des Pupillendurchmessers schliessen, selbst bei successiver Reizung der Augen durch Licht von so verschiedener Intensität, wie z. B. das Licht einer Gaslampe und das auffallende Sonnenlicht. Andererseits kann man bei operirten Kaninchen, bei denen die Operation eine merkliche Herabsetzung der Contractilität der Pupille im Gefolge hatte, nicht selten eine relativ grosse Bewegung der Iris des einen oder beider Augen constatiren. Ueberhaupt ist uns unbekannt, bis zu welchem Grade für die Contraction der Pupille die active Thätigkeit des Sphincter iridis, $d$. h. die Erregung des Oculomotorius, verantwortlich zu machen sei, und inwiefern jene Contraction eine passive Erscheinung - Zusammenfallen der Iris in Folge von Erschlaffung des Extensor iridis respräsentirt. Da sich nun unsere Operation auf eine Zerstörung der Bahn erstreckt, die vermuthlich den Reiz vom Opticus auf den Oculomotorius überträg't, so mussten wir bei unseren Beobachtungen die Thätigkeit des Extensors in Anrechnung bringen und konnten uns daher nur auf solche Veränderungen der Pupille beziehen, für die mit Sicherheit der Oculomotorius verantwortlich gemacht werden konnte. Dies ist aber jedesmal möglich, wenn die Pupillenverengerung einen grösseren Grad erreicht, als er dureh äusserste Erschlaffung des Extensors allein zu Stande kommt. Wir mussten daher zunächst die Wirkungsweise des Extensors für sich allein untersuchen.

$\mathrm{Zu}$ diesem letzteren Zwecke machten wir eine intracranielle Durchschneidung des Oculomotorius bei einem Kaninchen nach vorhergegangener Bestimmung des Durchmessers der Pupillen bei Reizung der Augen mit Gaslicht $\left(5 \frac{1}{2} \mathrm{~mm}\right)$ und mit Sonnenlicht (3 mm). Nachdem sich das Kaninchen von der Operation erholt hatte, unterwarfen wir es folgender Untersuchung: man setzte dasselbe in ein von der Sonne beleuchtetes Zimmer in der Weise, dass das Sonnenlicht vollkommen unbehindert in das Auge derjenigen Seite hineinfiel, auf der die Durchschneidung des Oculomotorius 
ausgefuhrt war; dabei erwies sich der Pupillendurchmesser des untersuchten Auges gleich $71 / 2$ mm; hierauf brachte man das Thier in einen ganz dunklen Raum und hier wurde in das nämliche Ange das Licht einer Gaslampe mit Hülfe einer planconvexen Linse geworfen; dabei erwies sich der Pupillendurchmesser gleich $10 \mathrm{~mm}$. Die Section, welche am anderen Tage nach der Operation gemacht wurde, ergab eine vollständige Durchschneidung des rechten Oculomotorius.

Demnach ergiebt sich, dass Bewegung der Iris auch bei vollkommener Paralyse des Oculomotorius bestehen kann. Allein in diesem Falle wird dieselbe durch die Thätigkeit des Extensor iridis bedingt und zwar durch Zusammenziehung und Erschlaffung des letzteren. Die Zusammenziehung findet bei Abnahme der Intensität des in's Auge fallenden Lichtes statt, die Erschlaffung dagegen bei Reizung des Auges durch Licht von grösserer Intensität. Ferner erfahren wir aus dem nämlichen Versuche, wie gross der Pupillendurchmesser des Auges bei einem Kaninchen mit vollkommener Paralyse des Oculomotorius ist. Derselbe misst $71 / 2 \mathrm{~mm}$, während er vor der Operation $3 \mathrm{~mm}^{1}$ ) betrug; er ist also durch die Durchschneidung des Oculomotorius um $41 / 2 \mathrm{~mm}$ grösser geworden.

Der beschriebene Versuch erlaubt uns in Bezug auf unsere Operationen folgende Schlussfolgerung zu machen. Wenn nach der Operation eine Vergrösserung des Durchmessers der engsten Pupille um ca. $4 \frac{1}{2} \mathrm{~mm}$ beobachtet wird, so dürfen wir mit Sieherheit voraussetzen, dass der Oculomotorius durch den Lichtreiz unerregt bleibt, für die Irisbewegung, die hierbei beobachtet wird, ist dann der Extensor iridis allein verantwortlich zu machen. In dem Falle aber, wo der Durchmesser der engsten Pupille nach der Operation weniger als um $4^{1} / 2 \mathrm{~mm}$ grösser geworden ist, nehmen wir eine Herabsetzung der reflectorischen Erregung des Oculomotorius an; für die Iriscontraction machen wir dann also neben der Extensorbewegung auch die active Thätigkeit des Sphincter iridis verantwortlich.

Indem wir jetzt zu einer ausfübrlichen Analyse der operativen

1) Ich will hier daran exinnern, dass die bei den Messungen angeführten Zahlen nur als approximative zu betrachten sind; ein Fehler von $1 / 4-1 / 2 \mathrm{~mm}$ war bei meinen Untersuchungen nie ausgeschlossen. 
Fälle übergehen, bemerken wir, dass die der ersten Gruppe sämmtlich einen rein negativen Charakter haben. Für den kleinsten Durehmesser der Pupille nach der Operation ergaben sich ganz ähnliche Zahlen wie vor der Operation (vergl. Nr. 3, 4, 6, 13). Indessen haben für uns die Fälle dieser Gruppe deswegen eine grosse Bedeutung, da sie uns die Theile kennen lehren, deren Verletzung keine Herabsetzung der reflectorischen Erregung des Oculomotorius nach sich zieht. Es stellt sich dabei heraus, dass eine Verletzung des ganzen unteren (Nr. 4) und der hinteren Hälfte des oberen Vierhügels (Nr. 3), sowie eine Verletzung der Commissura mollis (Nr.6) obne jenen Einfluss auf die Pupillenreaction bleibt.

Die operativen Fälle der zweiten Gruppe zerfallen ibrerseits in zwei Abtheilungen. $\mathrm{Zu}$ der Abtheilung $A$ habe ich die Fälle gerechnet, wo die Operation eine Störung in der reflectorischen Pupillencontraction nach sich gezogen hatte. Allgemein charakteristisch für diese Fälle ist eine Herabsetzung der reflectorischen Erregung beider Oculomotorii, wenn auch nicht immer in demselben Maasse, wobei die Abnahme der Pupillencontractilität sowohl bei gleichzeitiger Reizung beider Augen durch Licht als auch bei Reizung eines Auges für sich zu Tage tritt. Das Maass der Herabsetzung der Erregbarkeit der Oculomotorii ist verschieden. So wurde der Pupillendurehmesser beim Kaninchen Nr. 14 nach der Operation nicht mehr als um $1 \mathrm{~mm}$ grösser, während er beim

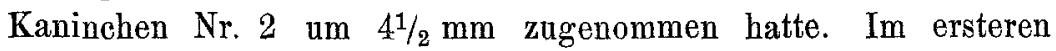
Falle können wir eine leichte Herabsetzung der reflectorischen Erregung der Oculomotorii annehmen, im letzteren eine vollkommene Unerregbarkeit derselben auf Licht.

Die wichtige Frage, die sich bei genauerer Analyse dieser Fälle aufdrängt, wäre etwa folgende. Welche Gehirntheile bedingen die besagte Veränderung in der reflectorischen Pupillenbewegung?

Die makroskopische Untersuchung der Gehirne hat ergeben, dass dieselbe in folgenden Fällen auftrat:

1. Bei Verletzung der hinteren Commissur in der Mittellinie (Nr. 14).

2. Bei Verletzung der hinteren Commissur seitlich von der Mittellinie (Nr. 9, 10, 15, 7).

3. Bei Verletzung der vorderen Hälfte des oberen Vierhügels in der Mittellinie (Nr. 2). 
Es durfte uns jedoch offenbar die makroskopische Untersuchung der Gehirne allein nicht die Berechtigung geben, die Erscheinung der besagten Störungen in der reflectorischen Irisbewegung auf eine Verletzung der hinteren Commissur zurückzuführen. Es mussten die Präparate einer mikroskopischen Untersuchung unterworfen werden.

Bei der letzteren stand die Entscheidung der Frage im Vordergrunde, ob nicht eine Verletzung der Kerne oder der Wurzeln des Oculomotorius vorhanden sei. Mit Ausnahme des Falles Nr. 15, wo in der That eine leichte Zerstörung des rechten Oculomotoriuskernes in Folge eines Blutergusses gefunden wurde, erwiesen sich in allen übrigen Fällen (Nr. 2, 9, 14, 10, 7) die Kerne sowohl als die Wurzeln des Oculomotorius ganz normal.

Eine andere nicht minder wesentliche Frage betrifft den Boden des dritten Ventrikels. Der letztere zeigt sich theils in der Mittellinie, theils seitlich von derselben lädirt in den Fällen Nr. 9, 14, 10, 7 (Fig. IV , 7, V, 7); allein andererseits in den Fällen Nr. 2 und Nr. 15, wo die Störungen in der reflectorischen Iriscontraction am stärksten ausgeprägt waren, erschien der Boden des dritten Ventrikels vollkommen intact (Fig. II). Wir sind demnach berechtigt zu schliessen, dass in den Operationen der Abtheilung A die Störungen in der reflectorischen Pupillenbewegung nicht durch eine Zerstörung des Bodens des dritten Ventrikels bedingt waren.

Diese Störungen können wir auch nicht von einer Zerstörung der Substanz des oberen Vierhigels, wie im Falle Nr. 2 (Fig. II), herleiten, denn in anderen Fällen (Nr. 9, 14, 15, 10) ist ein Defect in der Substanz des Vierhügels gar nicht vorgekommen (Fig. IV, V).

Dass die Verletzung der Commissura mollis (Nr. 9, 14) nicht als Ursache für die beschriebenen Störungen in der reflectorischen Pupillencontraction angesehen werden darf, geht aus dem hervor, was wir bei den Kaninchen Nr. 6 und 13 beobachtet haben.

Anlangend die Verletzung der hinteren seitlichen Wand des dritten Ventrikels, so kam dieselbe immer dann vor, wenn der Schnitt durch die hintere Commissur seitlich von der Mittellinie gegangen war, wie z. B. bei den Kaninchen Nr. 9 (Fig. VI, 2), Nr. 15 und Nr. 10 (Fig. V). Da aber bei den Kaninchen Nr. 14 und Nr. 2 (Fig. VI, 1), ungeachtet der scharf ausgesprochenen Herabsetzung der Erregbarkeit der Oculomotorii auf Licht gar keine Verletzung der hinteren seitlichen Wand des dritten Ventrikels stattgefunden 
hatte, so sind wir berechtigt zu schliessen, dass die Veränderungen in der reflectoriseben Pupillencontraction, welche bei den Kaninchen der Gruppe A auftraten, nicht von einer Verletzung der hinteren seitlichen Wand des dritten Ventrikels hergeleitet werden dürfen.

Es erïbrigt uns noch von den Folgen der Verletzung der hinteren Commissur zu sprechen. Dieselbe erwies sich bei mikroskopischer Untersuchung in allen Fällen, das Kaninchen Nr. 2 nicht ausgenommen, verletzt. Bei dem letzteren ergiebt die mikroskopische Untersuchung eine vollkommene Zerstörung der hinteren Commissur in ihrem hinteren Drittel (Fig. II). In den ibrigen Fällen war die hintere Commissur bald in der Mittellinie und dabei nur in ihrer vorderen Partie (Nr. 14), bald seitlich von der Mittellinie (Nr. 9; 10; Fig. IV, 6) zerstört, so dass ein Theil der Fasern (derjenige der anderen Seite) intact blieb.

Mithin sehen wir uns berechtigt, auf Grund dieser Untersuchungen der Abtheilung A die nach der Operation eingetretene Herabsetzung der Erregbarkeit der Oculomotorii auf Licht auf eine Verletzung der hinteren Commissur zurückzuführen und nicht ausschliesslich auf eine Verletzung irgend welcher anderer benachbarter Theile derselben.

Wir baben zur Abtheilung B zwei Fälle gerechnet, wo die Operation auch nicht ohne Einfluss auf die reflectorische Pupillencontraction geblieben war. Ein gemeinsamer charakteristischer Zug fuir diese Fälle ist die Vergrösserung des Pupillendurchmessers in einem Auge, welche nur bei unmittelbarer Reizung des betreffenden Auges durch Licht bemerkbar ist, während sich bei gleichzeitiger Reizung beider Augen beide Pupillen gleich weit und zwar nicht weiter als vor der Operation zeigen. Demnach handelt es sich hier um eine Herabsetzung der reflectorischen Erregung des einen Oculomotorius bei unmittelbarer Reizung des nämlichen Auges durch Licht.

Die Untersuchung der Gehirne schliesst in diesen Fällen eine Verletzung der hinteren Commissur aus und ergiebt eine Verletzung des binteren Abschnittes des Thalamus opticus und des Pedunculus conarii.

Da ich noch ein Mal zu den Fällen dieser Abtheilung zurückzukehren gedenke, so will ich hier nur noch bemerken, dass auch diese Fälle für uns nicht interesselos sind, indem sie lehren, dass 
Störungen im Bereich der reflectorisehen Pupillencontraction, welche für die Verletzung der hinteren Commissur charakteristisch sind, nicht bei der Verletzung von Theilen, die nach vorn von der hinteren Commissur gelegen sind, vorkommen.

III.

Nachdem wir die Erscheinungen im Bereich der reflectorischen Pupillencontraction kennen gelernt haben, welche bei einer beabsichtigten Durchschneidung der binteren Commissur auftreten können, wollen wir nun zur Entscheidung der Frage ibbergehen, welche Erklärung wir aus unseren bistologischen Untersuchungen herleiten können.

Der Lichtreiz, unter dessen Einfluss die Oculomotorii in Erregung gerathen, muss, um zu den letzteren zu gelangen, eine Bahn durchlaufen (Fig. I), die von den Fasern der Tractus optici $\left(d, d^{\prime}\right)$ und den Fasern der hinteren Commissur $\left(c, c^{\prime}\right)$ gebildet wird. Eine Verletzung, welche an irgend einer Stelle die Integrität dieser Bahn aufhebt, muss irgendwie auf die reflectorische Erregung der Oeulomotorii zurückwirken.

Betrachten wir zunächst den Fall, wo bei der Operation die Fasern des Tractus opticus, die zum Ganglion habenulae und zur Glandula pinealis verlaufen, verletzt werden und zwar nur auf einer Seite, d. h. einen Fall, wo der Schnitt in sagittaler Ebene nach aussen und parallel dem Ganglion habenulae geht. Gesetzt, es seien dureh den Schnitt die Fasern des Tractus opticus der linken Seite verletzt (Fig. I, 1). In diesem Falle gerathen die Oculomotorii nur unter dem Einfluss desjenigen Lichtreizes in Erregung, welcher durch den rechten Tractus opticus übertragen wird. Obgleich dieser Reiz von beiden Augen ausgeht, so ist doch klar, da der rechte Tractus opticus vornehmlich gekreuzte Fasern von dem linken Auge $A$ und eine relativ nur geringe Menge Fasern von dem rechten Auge $B$ führt, dass die Oculomotorii sich bei Beleuchtung des linken Auges während der Zeit, dass das rechte Ange verdeckt bleibt, unter einem Reiz von grösserer Intensität befinden werden als bei Beleuchtung des rechten Auges, während das linke verdeckt ist. Hieraus erhellt, dass die Pupille des linken Auges $A$ in dem Falle, wo das Licht direct in dieses Auge fällt und das rechte Ange $B$ verdeckt bleibt, sich mehr contrahiren muss als die Pupille des rechten Auges $B$ unter denselben Ver- 
hältnissen. - Ein ganz abweichendes Verhältniss werden wir an den Pupillen in dem Falle wahrnehmen, wenn dem Lichtreize nicht das zu untersuchende Auge, sondern das der anderen Seite ausgesetzt wird. In der That, reizt man das rechte Auge $B$ und beobachtet die Pupillencontraction des linken Auges $A$, so muss die Pupille dieses letzteren sich schwächer contrahiren als die Pupille des rechten Auges $B$ während der Reizung des Auges $A$, denn im ersteren Falle befinden sich die Oculomotorii unter dem Einflusse eines schwächeren Reizes als im letzteren Fall. - Bei gleichzeitiger Reizung beider Augen müssen wir eine gleichmässige Contraction beider Pupillen erwarten, denn der Lichtreiz wird von den Fasern des Tractus opticus auf die Fasern der hinteren Commissur tibertragen und von den letzteren in gleichem Maasse auf die beiden Oculomotorii.

Mithin ergiebt sich als Resultat, dass die Durchmesser der Pupillen gleich sein müssen, wenn das Licht gleichzeitig in beide Augen fällt. Aber die Pupille des Auges der der Läsion entgegengesetzten Seite muss bei directer Reizung dieses Anges weiter sein als bei der Reizung des anderen Auges und umgekehrt wird auf der Seite der Läsion die Pupille bei directer Beleuchtung enger sein, als wenn das. Licht nur in das andere Auge fällt.

Diese sämmtlichen Erscheinungen im Bereich der reflectorischen Irisbewegung bestanden bei den Kaninchen $\mathrm{Nr}$. 18 und 5 . Auf Grund derselben waren wir berechtigt zu erwarten, dass hier Fasern des Tractus opticus, welche zum Ganglion habenulae und zur Glandula pinealis verlaufen, lädirt worden wären. In der That ergab sich beim Kaninchen Nr. 18 eine Verletzung des hinteren Abschnittes des linken Thalamus opticus (Fig. VI, 4), d. h. eines Gebietes, wo eben hauptsächlich die Fasern des Tractus opticus verlaufen, die in frontaler Ebene uach dem Ganglion habenulae resp. der Glandula pinealis hinziehen, und beim Kaninchen Nr. 5 ergab sich eine Verletzung des Pedunculus conarii sinister (Fig. III, 3), d. h. eine Trennung des Fasersystems der binteren Commissur von den Fasern des linken Tractus opticus.

Eine Verletzung der Fasern der hinteren Commissur muss auf die reflectorische Iriscontraction ganz anders zurïckwirken als eine Verletzung der Fasern des Tractus opticus. Allein auch hier wird viel davon abhängen, ob die hintere Commissur total lädirt oder $a b$ nur ein Theil ihrer Fasern verletzt ist. 
Im ersteren Falle sind beide Oculomotorii für den Lichtreiz unzugänglich. Der letztere, von beiden Augen ausgebend, wird durch die Fasern der Tractns optici nur der Glandula pinealis und den Ganglion habenulae ïbermittelt, auf die Oculomotorii übertragen zu werden vermag er jedoch nicht, da der Zusammenhang zwischen diesen und der Glandula pinealis resp. den Ganglion habenulae - die hintere Commissur - vernichtet ist. Als Ergebniss der Operation müssen wir demnach vollkommene Unerregbarkeit der Oculomotorii anf Lichtreize erwarten, wie nach totaler Durebsehneidung beider Oenlomotorii (siehe oben). Die Pupillen müssen erweitert sein und zwar in demselben Maasse in beiden Augen, sowohl bei gleichzeitiger Beleuchtung beider Augen, als auch bei abwechselnder Beleuchtung derselben. - Kaninchen Nr. 2, bei dem, wie oben erwähnt, die mikroskopisehe Untersuchung eine vollkommene Zerstörung der hinteren Commissur ergeben hatte, bot nach der Operation in der That diejenigen Erscheinungen im Bereich der reflectorischen Irisbewegung dar, die bei Läsion der ganzen hinteren Commissur beobachtet werden muissen. Die Pupillen zeigten sich erweitert und zwar vermuthlich ad maximum (nach der Operation $71 / 2 \mathrm{~mm}$, vor der Operation $3 \mathrm{~mm}$ ); ihr Durchmesser war in beiden Augen derselbe and wechselte gar nicht, sei es, dass das Licht in beide Augen gleichzeitig fiel oder nur in eines derselben, während das andere verdeckt blieb. Kurz, beim Kaninchen konnten wir eine vollkommene Unterbrechung in der Uebertragung eines Reizes vom Tractus opticus auf die Oculomotorii und eine vollkommene Unerregbarkeit der letzteren auf Lieht constatiren; die Differenz in der Grösse des Durchmessers der Pupillen, welche eintrat, sobald das Gaslicht durch Sonnenlicht ersetzt wurde, führen wir, wie bereits oben erwähnt ist, nur auf eine Erschlaffung des Extensor iridis zurück.

Bei unvollständiger Läsion der hinteren Commissur in der Mittellinie (Fig. I, 3) wird die Zahl der auf beiden Seiten ausfallenden Fasern ungefähr gleich sein, so dass die beiden Bahnen der hinteren Commissur $c$ and $c^{\prime}$ auch nach der Operation ungefähr gleich bleiben. Hierans erhellt, dass ein Lichtreiz, welcher von den Fasern der Tractus optici tibermittelt wird, ungefähr in gleichem Maasse auf beide Oculomotorii iibertragen werden muss, allein unzweifelhaft mit geringerer Intensität als bei vollkommen intacter hinteren Commissur. Mithin ist als Ergebniss der Opera- 
tion eine Herabsetzung der Erregbarkeit beider Oculomotorii, also eine Vergrösserung des kleinsten Durchmessers der Pupillen beider Augen zu erwarten, welche sowohl bei gleichzeitiger Reizung beider Augen durch Licht als auch bei abwechselnder Beleuchtung eintritt. - Kaninchen Nr. 14 bietet uns das Beispiel einer solchen gleichmässigen Herabsetzung der Erregbarkeit der Oculomotorii auf Licht nach einer unvollständigen Läsion der hinteren Commissur in der Mittellinie. Wir betrachten hier die Herabsetzung der Erregbarkeit der Oculomotorii als gleich, trotzdem eine gewisse Differenz der Pupillendurchmesser vorhanden war, und zwar deshalb, weil bei diesem Kaninchen auch vor der Operation eine herabgesetzte Innervation der Iris des rechten Auges sich bemerkbar machte.

Es erübrigt uns noch die Frage zu erörtern, in welcher Weise eine unvollständige Läsion der hinteren Commissur durch einen Sagittalschnitt seitlich von der Mittellinie auf die reflectorische Iriscontraction wirken muss. In diesem Falle wird der Zusammenhang zwischen der hinteren Commissur und den Fasern des Tractus opticus der verletzten Seite gestört und dabei werden die Fasern der hinteren Commissur auf der der Läsion entgegengesetzten Seite in geringerer Anzahl durchtrennt als auf der anderen Seite. Angenommen, der Schnitt wäre links von der Mittellinie geführt (Fig. I, 2), so würden dadurch getroffen sein: 1. die Fasern des linken Tractus opticus, 2. ein Theil der Fasern der hinteren Commissur der rechten Seite $\left(c^{*}\right)$ und 3. die Mehrzahl oder alle Fasern derselben auf der linken Seite $(c)$. Als Resultat der ungleichseitigen Läsion der Fasern der hinteren Commissur muss sich eine verschiedene Uebertragung des Lichtreizes auf die beiden Oculomotorii ergeben. Im angenommenen Falle wird der linke Oculomotorius einer schwächeren Reizung ausgesetzt sein als der rechte. Dem entsprechend wird die linke Pupille (die der verletzten Seite) bei gleicher Beleuchtung beider Angen grösser als die rechte sein. Da sich nun die Läsion der hinteren Commissur gleichzeitig auf Fasern beider Seiten, wenn auch in verschiedenem Maasse, erstreckt, so ist als Resultat unserer Operation, abgesehen von der ungleichen Erregbarkeit der Oculomotorii, auch eine allgemeine Herabsetzung ihrer reflectorischen Erregung zu erwarten: die Durchmesser der Pupillen mïssen nach der Operation neben ihrer Ungleichheit noch vergrössert erscheinen. - Die Kaninchen 
Nr. 10, 9, 7 und 15, bei welchen, wie wir wissen, eine Verletzung der hinteren Commissur durch einen Sagittalschnitt seitlich von der Mittellinie vorhanden war, zeigten in der That eine Herabsetzung der Erregbarkeit der Oculomotorii auf Licht und dabei eine ungleichmässige Herabsetzung. So zeigte sich beim Kaninchen Nr. 10, bei welchem vornehmlich die linke Hälfte der hinteren Commissur verletzt war (Fig. V; VI, 2), die Pupille des linken Auges mehr erweitert als die rechte (rechtes Auge $3 \frac{1}{2} \mathrm{~mm}$, linkes Auge $4 \frac{3}{4} \mathrm{~mm}$ ), aber es war zugleich der Durchmesser der rechten Pupille grösser geworden, als er vor der Operation war (vor der Operation $2 \frac{1}{2} \mathrm{~mm}$, nach der Operation $3 \frac{1}{2} \mathrm{~mm}$ ). Dasselbe liess sich bei den Kaninchen Nr. 9 und 7 wahrnehmen.

Weil bei der eben beschriebenen Operation immer eine Trennung der Fasern der hinteren Commissur von den Fasern des Tractus opticus irgend einer Seite stattfindet, so ist klar, dass neben den eben beschriebenen Erscheinungen im Bereich der reflectorischen Irisbewegung wir auch die der Verletzung von Fasern des Tractus opticus der einen Seite bekommen müssen. Die Pupille des Auges der der Läsion entgegengesetzten Seite muss bei directer Reizung dieses Auges weiter sein als bei der Reizung des anderen Auges und umgekehrt wird auf der Seite der Läsion die Pupille bei directer Beleuchtung enger sein, als wenn das Licht nur in das andere Auge fällt. Diese Erscheinungen wurden in der That bei allen Kaninchen, wo eine Verletzung der hinteren Commissur seitlich von der Mittellinie stattgefunden hatte, wie z. B. bei den Kaninchen Nr. 7, 9, 10 und 15, wahrgenommen.

Die Veränderungen der durch Lichtreiz bedingten reflectorischen Irisbewegung, welche nach Läsion der hinteren Commissur auftreten, können wir schliesslich folgendermassen zusammenfassen.

Eine Läsion der hinteren Commissur hat immer eine Herabsetzung der Erregbarkeit beider Oculomotorii im Gefolge.

Die Grösse dieser Herabsetzung ist von den Dimensionen, welche die Verletzung der Fasern der hinteren Commissur erhalten hat, abhängig.

Eine vollständige Zerstörung der hinteren Commissur fuhrt zu einer vollständigen Unerregbarkeit der Oculomotorii, wie es bei gänzlicher Durchschneidung der Oculomotorii beobachtet wird.

Eine unvollständige ungleichseitige Läsion der Fasern der hinteren Commissur zieht eine ungleichseitige Herabsetzung der 
Versuche üb.d. Durchschneidung d. hinteren Gehirncommissur b. Kaninchen. 141

Erregbarkeit beider Oculomotorii nach sich: der Oculomotorius der Seite der grösseren Läsion ist weniger erregbar als der der anderen Seite.

Schliesslich ist es mir eine angenehme Pflicht, Herrn Prof. Goltz und Herrn Dr. Ewald für ihr liebenswtirdiges Entgegenkommen bestens zu danken.

\section{Erklärung der Abbildungen anf Tafel II.}

Fig. II. Frontalschnitt des Kaninchengehirns No. 2.

1. Hirnschenkelfuss.

2. C. genic. externum.

3. Aquaeductus Sylvii.

4. Tractus opticus.

5. C. quadrigeminum sup.

6. Commissura posterior.

Fig. III. Schema des Hirnstammes beim Kaninchen.

1. Thalamus opticus.

2. Schnittrichtang im Gehirn von Kaninchen No. 13.

3 .

4. Tractus opticus.

5. C. quadrigeminum Sup.

6. Schnittrichtung im Gehirn von Kaninchen No. 4.

7. C. quadrigeminum inf.

8. Schnittrichtung im Gehirn von Kaninchen No. 3.

9. Commissura posterior.

10. Ganglion habenulae.

11. Schnittrichtung im Gehirn von Kaninchen No. 6 .

Fig. IV. Frontalschnitt des Kaninchengehirns No. 9.

1, 2, 3, 4, 5, 6 dasselbe, wie in Fig. II.

7. Schnittstelle im Boden des dritten Ventrikels.

Fig. V. Frontalschnitt des Kaninchengehirns No. 10.

Die Bezeichnungen sind dieselben wie in Fig. IV.

Fig. VI. Schema des Hirnstammes beím Kaninchen.

1. Schnittrichtung im Gehirn von Kaninchen No. 2.

2.

3.

4.

$\begin{array}{lllll}n & n & n & & \text { No. } 9 . \\ & " & n & & \text { No. 14. } \\ & \Rightarrow & n & & \text { No. 18. }\end{array}$


Fig. 1
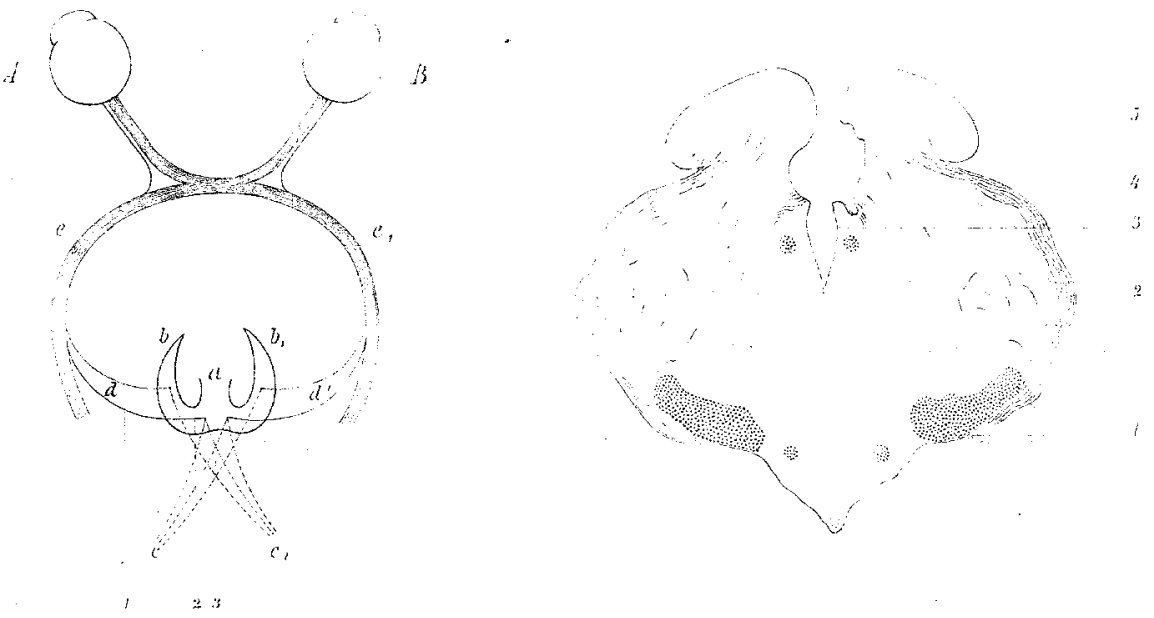

$\operatorname{lig} 3$
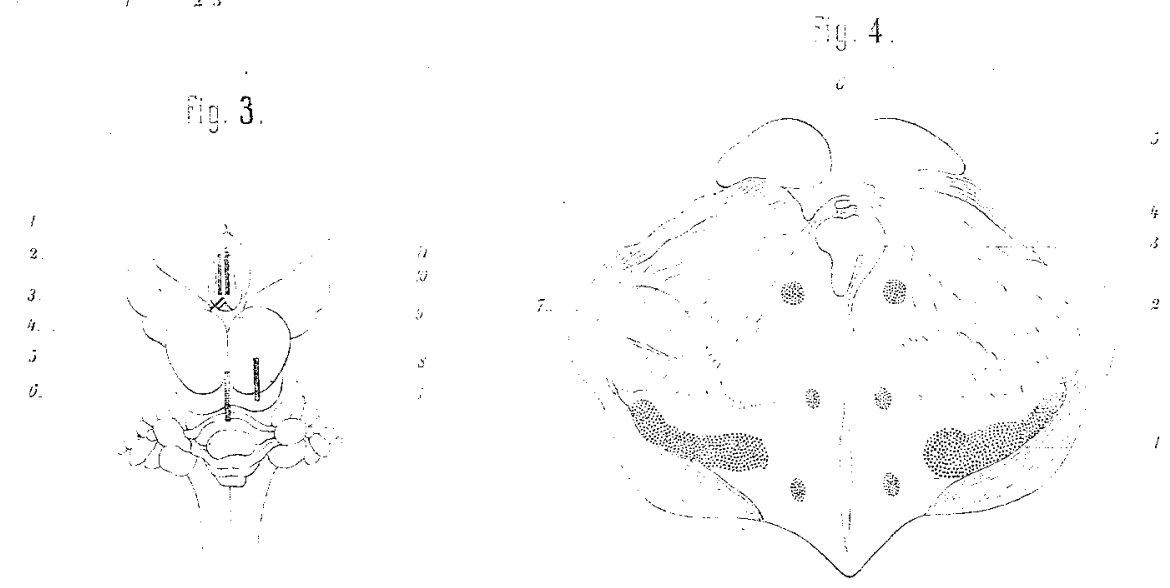

$\lg .5$

7.5

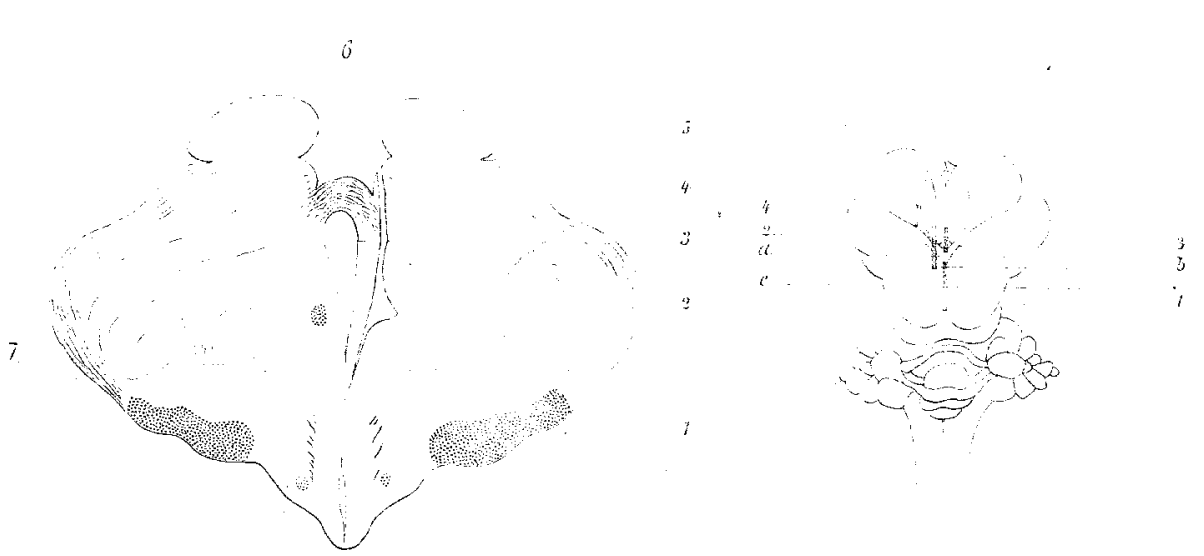

